$\begin{array}{ll}\text { NAL PROPOSAL NO. } \frac{8}{\text { Correspondent: }} & \begin{array}{l}\text { L. G. Pondrom } \\ \text { Department of Physics } \\ \text { University of Wisconsin } \\ \text { Madison, Wis. } 53706\end{array} \\ \text { FTS/Commercial } & 608-262-2284\end{array}$

\title{
Experiments in a Neutral Hyperon Beam
}

R. H. March and L. G. Pondrom University of Wisconsin

O. E. Overseth University of Michigan

June, 1970 
Experiments in a Neutral Hyperon Beam

\author{
R. H. Parch and L. G. Pondrom* \\ University of Wisconsin
}

\author{
O. E. Overseth \\ Iniversity of :ichigan \\ June 1970
}

ABSTRACT

We propose a survey experiment for a neutral hyperon beam, to measure production of $\Lambda^{\circ}, \bar{\Lambda}^{\circ}, \equiv^{0}, \Xi^{\circ}, K_{1}^{\circ}-K_{2}{ }^{\circ}$ near zero mrad. by $200 \mathrm{GeV}$ protons on complex nuclei. The detector will be sensitive to polarization of the hyperons. The same annaratus will then be used to search for $\equiv^{\circ} \rightarrow \mathrm{pm}^{-}$and to measure $\Lambda^{\circ}$ and $\bar{\Lambda}^{\circ}$ total and diffractive elastic cross sections in hydrogen.

${ }^{*}$ Correspondent 
Pace ?

I. NeutraI Hyperon Beam Survey

It has been known for some time that a practical neutral hyperon bean could be built at the National Accelerator Lahoratory. Many features of this beam were considered in some detail during the 1969 summer study. ${ }^{1}$ A charged particle spectrometer is described in this proposal which would be suitable for measurement of the relative fluxes in the neutral beam and for some early simple experiments. The experiments considered in detail are a search for the $\Delta S=2$ weak transition $\equiv^{\circ} \rightarrow p \pi^{-}$ and a study of $\Lambda^{\circ}$ cross sections in hydrogen in the $60 \mathrm{GeV}-130 \mathrm{GeV}$ energy range. The apparatus required for these experiments is well within the limits of existing technology, and can be constructed by June, 1971. It is assumed that the short lived neutral beam will share a common facility with the short lived negative beam, and that the experimenters will be responsible for the compatibility. A high ?mree of collaboration between the laboratory and the neutral and negative beam groums is necessary to insure success of the program and to place minimum demands on the accelerator.

Production of $\Lambda^{\circ}$ hyperons by $200 \mathrm{GeV}$ protons in hydrogen has been calculated by Walker ${ }^{2}$ using the formulas of Hagedorn and Ranft. Production of hyperons in complex nuclei has been considered by largolis and Pilkuhn. ${ }^{3} \Lambda^{\circ}$ hyperon production is predicted to reach approximately $3 \Lambda^{\circ}$ per sterad per $\mathrm{GeV} / \mathrm{c}$ interacting nroton at $0 \mathrm{mrad}$ and $120 \mathrm{GeV} / \mathrm{c}$. Since the decay length for $120 \mathrm{GeV} / \mathrm{C} \Lambda^{\circ}$ hyperons is 7.8 meters, the distance between the production target and the experimental area must be kept short, the order of 10 moters. 


\section{Page 3}

The main problem in the design of the short lived bean is the matching of this requirement to the shielding necessary for the oneration of electronic detectors. Ten meters of iron would furnish a satisfactory hadronic shield, and the muons could be bent way magnetically, giving a muon free corridor for the apparatus. A curved channel could be cut in the shield to accommodate a negative hyperon beam. A. $200 \mathrm{GeV}$ proton beam in the intensity range $10^{8}-10^{10}$, suitable for production of useful hyperon fluxes, has been discussed for Experinental Area Two by J. Walker, R. Stefanski, and A. Roberts. ${ }^{4}$ Figure 1 shows a oroposed layout from the proton target to the experimental decay region.

Estimates of the expected neutral particle yields based on Walker's calculations are shown in Table I. These numbers indicate that a beam survey could be performed with as few as $10^{8}$ interacting protons/nulse. Figure 2 shows the apparatus for the beam survey. The basic idea is to measure the invariant mass of two oppositely charged particles from two body decays of neutrals. The design mass resolution of the spectrometer will be $\pm 1 \%$. The spectrometer magnet pole face should be about $30^{\prime \prime}$ wide with an $8^{\prime \prime} \mathrm{gap}$ and a BL of $1800 \mathrm{~kg}$-inches. Magnets with these characteristics are not uncomnon at presently onerating accelerators. For example an Argonne Labs BM-109 (24-8-72) could be used. The experimenters will be responsible for obtaining the magnet.

Great care must be taken in the construction of the spectrometer to keep the amount of material presented to the neutron beam to a minimu, and to keep the sensitivity of the apparatus to charged particle background as low as possible. No scintillators will be used in front of the magnet - the trigger will employ three "Charpak" counters. The 
largest of these will be about $3 n \mathrm{~cm} \times 30 \mathrm{~cm}$. Sach counter will be $2.5 \mathrm{mg} / \mathrm{cm}^{2}$ thick, nresenting about $4 \times 10^{-5}$ of a neutron interaction length, or about $10^{4}$ neutron counts/pulse in the third counter at full bean intensity. This should be a manageable counting rate. The large chambers behind the magnet will not be sensitive in the neutral beam region. The muon free "corridor" will cover all counters provided the spectrometer magnetic field and the magnetic field in the hadron shicld are normal to each other.

Instantaneous data collection rates of the order of 500 per second during the bearn spill will be buffer stored, and analyzed between accelerator pulses. When surveying the $\Lambda^{\circ}$ and $\mathrm{K}_{\mathrm{s}}^{\circ}$ components of the beam, 2 considerably reduced proton beam intensity could be used. For the $\equiv^{\circ}$ studies, however, an incident flux of $10^{9}$ protons on target would be convenient. At 1000 pulses/hour, our data capability wculd be roughly $10^{6}$ triggers per day.

Table II shows the expected counting rates for a 10 meter decay length using the fluxes in Table $I$ and the detector geometry of Fig. 2. The majority of decays will be approximately equal numbers of $\Lambda^{\circ} \rightarrow p \pi^{-}$ and $\mathrm{K}_{S}^{\circ} \rightarrow \pi^{+} \pi^{-}$. About $10 \%$ of these decays will fit either invariant mass because of the finite mass resolution of the system and the unknown beam momentum. This will nresent no problem for the yield measurements because the background will subtract easily from the peak, but the identification of a particular decay will be uncertain $10 \%$ of the time. This ambiguity can be resolved by the addition of a six meter long hydrogen gas cherenkov counter behind the magnet. This counter will serve to reject $p^{\prime} s$ and $\bar{p}$ 's with momentum below $80 \mathrm{Ge} / / \mathrm{c}$, and will 
aid in measuring the yields of the other less copious neutral components of the beam. The reconstruction of the $\Lambda^{\circ}$ events will allow a measurement of $\alpha^{\Lambda} \overline{\mathrm{P}}$, and hence the $\Lambda^{\circ}$ polarization. To search for a non zero value of $\overline{\mathrm{P}}$, data will be taken varying the incident proton beam angle, since it is expected that the polarization will have a very steep anpular dependence. A downstream steering arrangement for the protons consisting of two main ring magnets would permit a study over a $10 \mathrm{mrad}$ range. The decay sequence $\Xi^{\circ}+\Lambda^{\circ} \pi^{\circ}, \Lambda^{\circ} \rightarrow \mathrm{p}^{-}$will be detected by a coincidence between a $\Lambda^{\circ}$ decay and a $\gamma$ ray conversion from the $\pi^{\circ}$. A $\gamma$ counter telescope will be placed behind the apperatus for this purpose. Aside from accidental coincidences the only backoround for this measurement will be $\pi^{\circ}$ production by $\Lambda^{\circ}$ 's in the beam, which should be a few percent of the expected $\equiv^{\circ}$ yield. The flux of $\bar{\Lambda}^{\circ}$ 's will be measured by using the gas cherenkov counter to veto $\pi^{-}$mesons, and reconstructing events assuming the charged particles to be $\left(\pi^{+} \bar{p}\right)$. If the gas counter is $98 \%$ efficient for $\pi^{-}$, then the $\mathrm{K}^{\circ}+\pi^{+} \pi^{-}$triggers will be a factor of 100 more numorous than the $\bar{\Lambda}^{\circ}$ triggers, but only $10 \%$ of the $\mathrm{K}^{\circ}$ 's will reconstruct near the $\bar{\Lambda}^{\circ}$ mass. The anti-hyperons will then be a $10 \%$ signal on a background which is slowly varying in invariant mass. In this case a statistically significant $\bar{\Lambda}^{\circ}$ yield of several thousand events should be obtained in a day's run at $10^{8}$ protons/pulse. 
II. Searcl: for $\equiv^{\circ} \rightarrow n \pi^{*}$.

Toar locays in wich $\Delta S=2$ have not been observed at rates cormatible with first order in the weal counling constant f. The ${ }^{K^{\circ}}{ }^{\circ}-K_{L}^{0}$ mass difference is renerated by a $\Delta S=2$ transition, but is consistent in mapnitude with a socnd ordar wak internction, since $\delta n \sim r_{s}$, wich is proportional to $G^{2}$. However, terms in the weak Lagrangian which contribute to om in first order must be even under charge conjugation, so it is possible that $\Delta S=2$ transitions with branching ratios of $10^{-3}-10^{-4}$ exist which are ord under charge conjupation. ${ }^{5}$ The observed $\mathrm{CP}$ violation could he one manifestation of such terms. There are not very many strange particle decays for wich $\Delta S=2$ cliannels are availaile. The present experimental limits on the transitions $\equiv^{-} \rightarrow n \pi^{-}$and $\equiv^{0}+n \pi^{-}$are eac $\lesssim 1 n^{-3} \cdot 6$ This exporiment is dosigned to pust the limit to $\sim 10^{-6}$.

Two modifications of the spectrometer srstem will be required to search for $\equiv^{0} \rightarrow \mathrm{p}^{-}$. The decay distance will be shortened to six meters and the magnet gap shimed to $10^{:}$to incroase the apertures because of the ligh transvers 3 momentum of the decay. A ring scintillation counter will be insorted in front of the magnet to discriminate against protons from $\Lambda^{\circ} \rightarrow \mathrm{pm}^{-}$wicl will be closer to the neutral bcam. About $12 \%$ of the $\equiv^{\circ} \rightarrow \mathrm{pm}^{-}$protons will be lost by this requirement, and $98 \%$ of the $\Lambda^{\circ}$ decays rill bo rejected. Thus ahout $3 \times 10^{6}$ triggers whic'? will in fact be $\Lambda^{\circ} \rightarrow \mathrm{pm}^{-}$will be recorded and reconstructed in order to have "looked at" $10^{6} \equiv 0$. This should take about 72 hours of runing at $10^{9}$ protons/pulse, and a data rate of 500 events/pulse. 
III. $\Lambda^{\circ}$ Cross sections in . viropor.

The existing data on $\Lambda$ interactions ave recently been compiled by the Particle Data Groun. ${ }^{7}$ The data extend up to $4 \mathrm{GeV} / \mathrm{c}$, tut are quite meager above $.5 \mathrm{GeV} / \mathrm{c}$. The total cross section in the GoV region is roughly $40 \mathrm{~m}$, half of wich is elastic. The $\Lambda p$ systen appoars to have no direct channel resonances, and is similar to $p$ except for the absence of the one pion exchange term in elastic scattering. It sems reasonable to use the pp system as a guide in estimating $\Lambda p$ cross sections. Serpulhov data for pp scatterirg indicate a total cross scction $\sigma_{T}=39$ millibam and a diffraction elastic cross section of the form $\frac{d \sigma}{d t} \propto e^{b t}$ there $b=(7.0+1 n s) \mathrm{GeV}^{-2} .^{8}$ The measuroment of $\Lambda p$ total and diffraction elastic cross sections in the region $60 \mathrm{rov}-130 \mathrm{GoV}$ would clearly Data for both cross sections can be talen simultaneously.

The experimental arrangemont will be identical to that for the bean sumve except for the insertion of a one meter long linuid hydrogen target in the upstroam part of the ton meter decar nat?. To detect proton recoils from diffraction scattering a range teloscone will surround the target in $72 \pi$ azimuth configuration, and will be sonsitive to protons with energies between about $40 \mathrm{seV}$ and $250 \mathrm{MoV} . \mathrm{A} 40 \mathrm{MeV}$ proton corresponds to a momentum transfer $t=2 \mathrm{NT}=.08(\mathrm{GeV})^{2}$, and a scattering angle $\theta=3 \mathrm{mrad}$ for a $100 \mathrm{GeV} \Lambda^{\circ}$, wich will bo approximatcly the $e^{-1}$ point in the diffraction cross section if the parameter b $12(\mathrm{Gol} /)^{-2}$. Smaller momontum transfors will be difficult to detect by a coincidence method, so an extranolation tecinique will be required to obtain $\frac{d \sigma}{d t}(t=0)$. Tho total cross section will bo measured by a 
transmission mothor. The intorent widt of the hom defined thy the collimator will te 0.6 mrat fum with periens vings from collinator halo and scattering in the mpty taret. With the target full the dj.ffraction scattering will contribute to the wings, as will $\Lambda^{\circ}$ production by the $\mathrm{K}_{L}{ }^{\circ}$ and $\mathrm{n}$ components of the boam. Since these effects will create $\Lambda^{\circ}$ 's aprreciably outside of the inherent bean width, tho subtraction of the "background' from the undeflected hyneron beam should be possible.

Thile the neutrons should nresent no prohlon through pro uction of $\Lambda^{\circ} \mathrm{s}$, they will create an intense charged particle background wich will limit the usealle flux in the noutral knam. The ratio in Table I for $\mathrm{n} / \Lambda^{\circ} \sim 200$; assuming overy netron produces one $\mathrm{c}^{\mathrm{r}}$ arged particle in one meter of hydrogen, a maximum neutron intensity of $\sim 5 \times 10^{4}$ per pulse, or $2301 \%$ pulse vill bo required to keep the accirentals rates mangeable. If $\sigma_{\text {tot }} \sim 40 \mathrm{mb}, 13 \%$ of the $\Lambda^{\circ}$ 's will interact in one meter of hydrogen, so the statistical error in the difference measurement will be $\sim \frac{11}{\sqrt{N^{T}}}$ where $N$ is the flux of unscattored lyperons. Thus 200 $\Lambda^{\circ} /$ pulse will give $2 \times 10^{6} \Lambda^{\circ}$ per day, or a statistical error of a few percent in $\sigma_{\text {tot }}$ in the $80 \mathrm{GeV}$ to $120 \mathrm{GeV}$ region where most of thr detected $\Lambda^{\circ}$ flux will bo concentrated. Increasing the $\Lambda^{\circ}$ production angle at the proton target from near 0 to $5 \mathrm{mrad}$ or even $10 \mathrm{mrad}$ will decrease the $n / \Lambda^{\circ}$ ratio at production, but $t^{*}$ is improvemont will be lost in the a meter flight path through the shield because the $\Lambda^{\circ}$ momentum spectrum shifts to lower momenta as the production angle is increasod. The measurement of $\vec{\Lambda}^{\circ}$ in total cross sections may also te feasible. The use of a 10 mrad production angle for the neutral bean would be advantagcous in this case, because the antiparticle momentum spectrum 
should not shift dowmerr shamly compared to ? mrad. Thus the $n / \bar{\Lambda}^{\circ}$ ratio sould be $\approx 2 \times 10^{4}$ at 10 mrad, rather than $2 \times 10^{6}$ moted in Table $T$. Using the accidentals limits quoted aboro, a maximm bcam of a far $\bar{\Lambda}^{\circ}$ per nulse should be possible, requiring the order of $15^{9}$ protons/pulse on target. Since the cross sections for $\hat{\Lambda}^{\circ} \mathrm{p}$ will he larger than the $\Lambda^{\circ} \mathrm{p}$ cross sections, measurements of $\sigma(\vec{\mu} \mathrm{p})$ to $19 \%$ could be obtained in the order of one week of running. 
IV. Sumbary - Poquiroments for the Experinent.

The following requirments will be placed on the accelerator facilities:

1.) A proton bean near $200 \mathrm{GeV}$ wit? a slow spill and an intensity variable between $1 n^{8}$ and $10^{9}$ protons/pulse. Steering magnets to vary the incident proton angle at the hyperon production target over the range 0-10 mrads would be advantagecus. This requires tro min-ring mapicts.

2.) A neutral channel of approximately one microsterradian solid angle in a magnetized hadron shield 10 moters $10 n$,

3.) Floor area for the abnaratus roughly 35 noters lone by 3 meters wide by 3 meters irjgh.

4.) A. 1 meter cylindrical indrogen target $2.5 \mathrm{~cm}$. in dianeter.

5.) Tota1 number of beam protons interacting in the target:

a.) $6 \times 10^{12}$ protons at $10^{8} /$ pulso for tuning and boam survey ( 3 days):

b.) $10^{13}$ protons at $3 \times 1 n^{8} /$ mulso for the $\equiv^{0}+\mathrm{pm}^{-}$ $\operatorname{search}(3$ dxys) ;

c.) $7 \times 10^{12}$ protons at $5 \times 1 n^{7} /$ pulse for the $\Lambda^{\circ} \mathrm{p}$ and $\bar{n}^{\circ} \mathrm{p}$ scattering measurements ( 1 weel). 
The following epparatus will bo furnisher sy the experimenters:

1.) Chanicers, counters anr associated electrenics.

2.) Yim snced buffer storage register.

3.) Spectromoter magnet with a gap approximately 39" wide

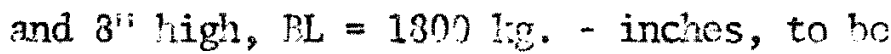
borrowed or built on a cost shared basis. The total cost should bo about $\$ 8 n_{8}, 900$.

4.) On line computor in the ASI $60-40$ class (24 bit words, $32 \mathrm{k}$ menory). The commuter fecility will be shared with tho negativo hyperon oxperiment. 
TABLE I

Neutral particle fluxes estimated using Walker's ${ }^{2}$ yield curves. A solid angle of $10^{-6}$ sterad at $0 \mathrm{mrad}$ and a flight path of 8 meters were assumed. All fluxes are per $10^{10}$ interacting nrotons.

\begin{tabular}{|c|c|c|c|}
\hline Particle & Flux & $\begin{array}{l}\text { Most Probable } \\
\text { Momentum }\end{array}$ & \\
\hline $\mathrm{n}$ & $10^{8}$ & 180 & $\begin{array}{l}\text { Slowly varying momentum } \\
\text { Spectrum } 100-200 \mathrm{GeV} / \mathrm{c}\end{array}$ \\
\hline$\overline{\mathrm{n}}$ & $10^{5}$ & 40 & $\begin{array}{l}\text { Anti-particle momentum } \\
\text { Spectrum falls off sharpl } \\
\text { with increasing momentum? }\end{array}$ \\
\hline$\Lambda^{\circ}$ & $5 \times 10^{5}$ & 120 & \\
\hline $\bar{\Lambda}^{\circ}$ & $50 \quad ?^{\mathrm{a}}$ & 40 & \\
\hline$\equiv^{\circ}$ & $5 \times 10^{3} ?^{b}$ & $120 ?$ & $\begin{array}{l}\text { Same momentum spectrum } \\
\text { Assumed for } \Xi^{\circ} \text { and } \Lambda^{\circ}\end{array}$ \\
\hline$\Xi^{\circ}$ & .5 & 40 & \\
\hline $\mathrm{K}_{\mathrm{L}}$ & $1.35 \times 10^{6}$ & 60 & interference \\
\hline$K_{S}$ & $.15 \times 10^{6}$ & $80 J$ & int ot \\
\hline
\end{tabular}

a) Antiparticle/particle ratio assumed to be $10^{-3}$. The extra factor of .1 comes from the decay of the $\bar{\Lambda}^{\circ}$ 's which have an average momentum $1 / 3$ that of the $\Lambda^{\circ} \mathrm{s}$.

b) The $\equiv$ flux is rather arbitrarily taken to be $1 \%$ of the $\Lambda^{\circ}$ flux. 


\section{TABLE IT}

Neutral particles detectei in a 10 meter decay 1ength. These numbers were obteined from the fluxes and momontum spectra in Table $I$, combined with the appropriate lifetimes, branching ratios, and detection efficiencies. All yields are for $10^{10}$ interacting protons.

\begin{tabular}{|c|c|c|}
\hline PARTICLE & $\begin{array}{l}\text { FLUX \&M } \\
\text { FROM TGT }\end{array}$ & $\begin{array}{l}\text { YIEIS Fam } \\
8 \text { to } 18 \mathrm{M} \\
\end{array}$ \\
\hline$\Lambda^{0}$ & $5 \times 10^{5}$ & $2.6 \times 10^{5}$ \\
\hline $\bar{\Lambda}^{\circ}$ & 50 & 26 \\
\hline$\equiv^{0}$ & $5 \times 10^{3}$ & $1.3 \times 10^{3}$ \\
\hline$\Xi^{0}$ & .5 & .1 \\
\hline$K_{S}$ & $1.5 \times 10^{5}$ & $1.0 \times 10^{5}$ \\
\hline$K_{L}$ & $1.35 \times 10^{6}$ & $2 \times 10^{3 !}$ \\
\hline
\end{tabular}

a.) For illustration, the efficiency of .26 for $\equiv^{\circ}$ comes from .33 for the chain $\equiv^{\circ}+\Lambda^{\circ}+\mathrm{pm}^{-}$to be detected in 10 meters, and .8 for detecting a $\pi^{\circ} \gamma$ ray behind the anparatus.

b.) This number includes leptonics and $\tau$ 's wich count but do not bave unique invariant mass. For $\mathrm{K}_{\mathrm{L}}^{\mathrm{O}} \rightarrow \pi^{+} \pi^{-}$, the exoected yie1d is 210 . 


\section{IISTE CanTIOE}

1.) Possible shieli and collinator confimuration for the short lived beams. The letters refer to the following: A) incident nrotor beam in the intensity range $10^{8}-10^{9}$ wits variable angle of incidence over $0-1 n$ mrad; B) one interaction length $\mathrm{Cu}$ target; C) heavymet or other high density shiold near the target with a channel in the forward direction; D) one microstorad channel in the irm shield, 8 meters long: and E) mapnetized iron shield. The heavymet high density shield is solid for about $1 / 2$ interaction length in the direction of the noutra1 bean to eliminatc the $\gamma$ ray component.

2.) Proposed experimental arrangement for the beam survey. A vacuum pine will be placed along the 10 meter decay length during tho survey to minimize gas interactions. P1, P2, P3 are proportional counters of the Charpak tync. "Is the analyzing mapnet. $s 1,82, s 3$ are magnetostrictive readout wire chmbers. $C$ is a 6 meter $1 \mathrm{mg}$ hydrogen gas Cherevlov counter snlit into two separate corpartments for positively and negatively charged particles. H1 and H2 are scintillation counter hodoscopes, and $\mathrm{H3}$ is a lead and lucite hodoscopo for $\mathrm{y}$ ray conversion. For the cross section studies a 1 meter long liquid hydrogen target will be inserted at $\mathrm{L}_{2} \mathrm{~L}_{2}$. 


\section{PFFEDENICFS}

1. R. F. Warch, Nationa1 Accelerator Laboratory, 1969 Sumerer Study, SS-69-3, Vo1. I, p. 173 (unpublished).

2. T. G. Walker, National Accelerator Labcratory, 1968 Sumer Study, B. 5-68-24, Vo1. II, p. 59 .

3. B. Margolis and H. Pilkuhn, Karlsruke External Report, June, 1968. (umpublished)

4. J. K. Walker, R. Stefanski, and A. Poberts, NAL Internal P.enort, 1970 (umpublished)

5. L. B. Okn', Soviet Journal of Nuclear Physics 1, 806 (1965)

6. Dauber, Berge, Hubbard, Merrill, and Miller, Ptys. Pev. 179,1262 (1969)

7. Compilation of YN Reactions, Particle Data Groum UCRL - $20000 \mathrm{YN}$, 1970 (unpublished)

8. Beznogikh, et al. Phys. Letters $\underline{30 B}, 274$ (1969) 
13

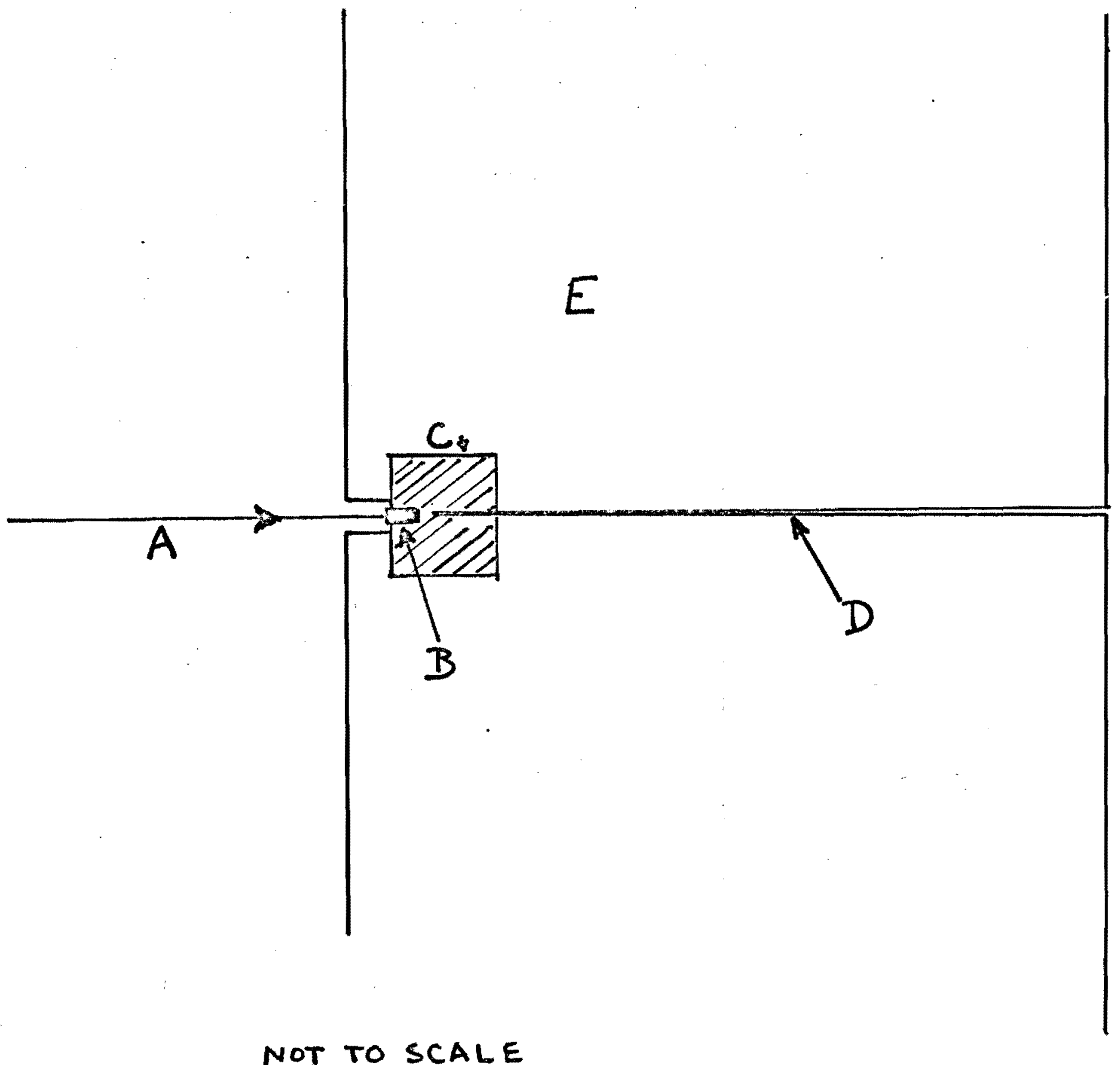

NOT TO SCALE

FIG 1 


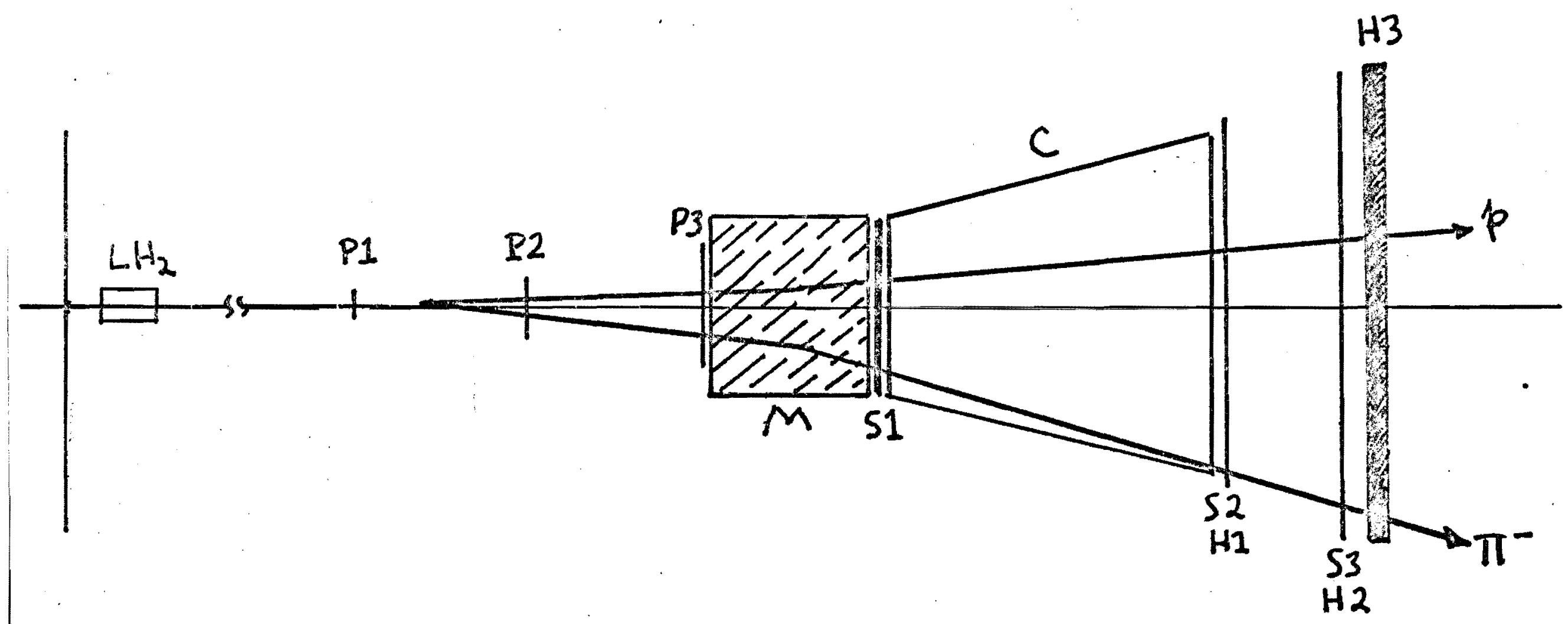

SCALE

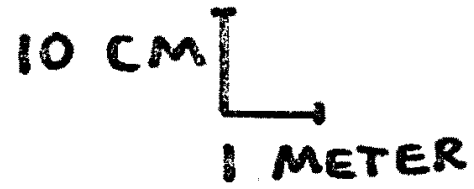

FIG 2 